\title{
Development and Evaluation of a New
}

\section{Questionnaire to Assess Social Cognitive Factors of Self-Management in Patients with Type 2 Diabetes: A Psychometric Study}

\section{Hamed Mirzaei}

Isfahan University of Medical Sciences https://orcid.org/0000-0002-5330-7749

Mansour Siavash

Isfahan University of Medical Sciences

Hossein Shahnazi

Isfahan University of Medical Sciences

Ahmad Ali Eslami ( $\sim$ eslamiaa@gmail.com)

Isfahan University of Medical Sciences

\section{Research}

Keywords: Factor analysis, SCT, Self-Management, Diabetes, Psychometric, HbA1c

Posted Date: November 2nd, 2021

DOI: https://doi.org/10.21203/rs.3.rs-1001057/v1

License: (c) (i) This work is licensed under a Creative Commons Attribution 4.0 International License.

Read Full License

Version of Record: A version of this preprint was published at Journal of Diabetes \& Metabolic Disorders on February 9th, 2022. See the published version at https://doi.org/10.1007/s40200-022-00999-0. 


\section{Abstract \\ Background}

Self-care behaviors are essential in controlling blood sugar and preventing mortality and complications of diabetes. The evidence suggests that social cognitive factors play crucial role in adopting self-care behaviors ..

\section{Objectives}

this study aimed to develop and examine the psychometric properties of the Diabetes Self-Management Questionnaire based on the social cognitive theory (DSMQ-SCT).

\section{Methods}

The present methodological cross-sectional study investigated 460 patients with T2DM referred to Isfahan Endocrine and Metabolism Research Centre, Isfahan, Iran using the convenience sampling method. We assessed the reliability (internal consistency), content validity, convergent validity and construct validity. Construct validity was evaluated using exploratory factor analysis (calibration sample $=230$ ) and confirmatory factor analysis (validation sample=230).

\section{Results}

In the EFA with the varimax rotation, we extracted four factors that could explain $65.9 \%$ of the total variance of the DSMQ-SCT scale. The CFA exhibited a satisfactory fit level for the four-factor structure extracted from the EFA with the following measures. CFI: .971, IFI: .971, NFI: .942, TLI: .966, RMSEA: .046). Internal consistency was good (Cronbach's alpha coefficient for the whole scale: 0.88; the subscales: 0.85 to 0.92 ). The convergent validity showed significant negative correlations between DSMQ-SCT subscales and HbA1c.

\section{Conclusion}

The DSMQ-SCT is a valid and reliable instrument to assess social cognitive factors associated with selfmanagement behaviors in patients with T2DM. Therefore, this instrument can be used in both future research and clinical practices.

\section{Introduction}


Diabetes mellitus (DM) is the most common and important chronic metabolic disorder in humans due to impaired insulin secretion, resistance to peripheral actions of insulin, or both. Currently, the disease is considered a major global public health issue owing to its high prevalence ${ }^{1}$. The International Diabetes Federation report indicates that there were 463 million patients with diabetes in the world in 2019, and it is estimated that by 2045 , the population of diabetic patients will rise to approximately 700 million people, mostly in low- and middle-income countries ${ }^{2}$.Iran, with 5.4 million adults (20-79 years) is the third country in terms of diabetes prevalence in the MENA region. Type 2 diabetes is the most common type of diabetes, accounting for almost $90 \%$ of all diabetes cases ${ }^{2}$. The patients with diabetes compared to others in the community are at higher risk of cardiovascular disease, stroke, peripheral vascular disease, premature death, disability, and low quality of life $e^{3}$. The disease imposes a significant financial burden on communities and the health care system ${ }^{4}$. The patient's responsibility to adopt self-care behaviors is critical in the effective control and management of diabetes ${ }^{5}$. The goals of diabetes management measures are to maintain blood sugar levels within the normal range, to prevent the disease complications, and ultimately to improve the patients' quality of life; the accomplishment of these goals totally depends on patients' adoption and consistent commitment to self-care behaviors ${ }^{6}$. Self-care behaviors in patients with diabetes include blood sugar self-monitoring, proper diet, exercising, adherence to medications, and foot care ${ }^{7}$. The results of a systematic review by Leonard et al. indicated that social support played key role in the self-care behaviors of patients with type 2 diabetes $^{8}$. In addition to social support, outcome expectations and outcome assessment are other factors influencing the self-care of diabetic patients ${ }^{9,10}$. Outcome expectations are the individual's belief in the likelihood of a consequence of a particular behavior, while outcome assessment is the importance and value that a person attaches to that outcome ${ }^{11}$. Self-efficacy is significant factor in various aspects of behavioral performance and according to studies, it is also a significant predictor of self-care behaviors in diabetes ${ }^{12,13}$. In fact, selfefficacy in most studies investigating the factors influencing self-care and diabetes control has been identified as not only an influential factor but also the most important and strongest one in this regard ${ }^{14}$. Social Cognitive Theory (SCT) is a useful behavioral theory to explain the self-management behaviors of patients with chronic diseases ${ }^{15}$. This theory aims to explain human behaviors as a triple reciprocal causality, and it considers behavior as the center of a triangle with individual factors, environmental factors, and behavioral factors as its three angles that are connected to each other in pairs ${ }^{11}$. Social support, outcome expectations, outcome expectancy and self- efficacy are the key constructs of the social cognitive theory that are relevant to diabetes self-management ${ }^{9,10}$.The limitations of existing diabetes self-care tools have been reported in recent narrative and systematic reviews as follows: (1). Most instruments are obsolete ${ }^{7}$. (2). Some of these instruments measure only one dimension of diabetes self-care behavior such as diet, physical activity, and blood sugar monitoring ${ }^{16}$. (3). None of the available instruments provides the evidence of a strong validation process (For example, in most cases standard validity is estimated using scores from other scales instead of clinical indicators) or does not present a theoretical framework to identify factors influencing self-care behaviors ${ }^{17}$. These reviews also emphasized the need for designing a theory-based instrument with strong psychometric characteristics 
for the self-management of patients with type 2 diabetes ${ }^{16,18}$.In addition, it was found that existing measures for Social Cognitive factors related to Self-Management had no focus on all behaviors or included only a construct of SCT.For example,the Diabetes Management Self-Efficacy Scale( DMSES) ${ }^{19}$ and social support scale for self-care in middle-aged patients with type II diabetes (S4-MAD) ${ }^{20}$ focus only on one construct of SCT.Based on a review of the texts a comprehensive theory-based instrument was not found that simultaneously measured the social cognitive factors associated with diabetes selfmanagement based on the social cognitive theory. Therefore, this study aimed to develop and evaluate a new instrument to assess the social cognitive factors of self-management in patients with type 2 diabetes based the social cognitive theory (DSMQ-SCT).

\section{Methods}

\section{Study design}

The present methodological study was conducted in two phases. In the first generation of items and development of the questionnaire were carried out. In the second phase, the reliability and validity of the instrument were evaluated.

\section{Setting and participants}

The present a cross-sectional study was conducted from June2019 to September 2019. A convenience sampling method was used to recruit patients with T2DM from the Diabetes Clinic of the Isfahan Endocrine and Metabolism Research Center (IEMRC), Isfahan,Iran. The minimum sample size required for factor analysis was 5 to 10 per item of scale ${ }^{21}$. A total of 460 patients with T2DM participated in the study.Inclusion criteria were as follows:(1) being at the age of 18 years or older;(2) being diagnosed with type 2 diabetes for at least 3 months;(3) being able to read and speak in Persian; (4) undergoing the $\mathrm{HbA1c}$ lab test in the past two months;(5) providing the consent to cooperate in the study. Patients were excluded if they had;(1)cognitive impairment;(2) serious diabetes complications( neuropathy, kidney disease, amputation, other complications); (3)incomplete questionnaires.

\section{Ethics approval}

The participants were informed about the study and their written informed consent were obtained. This study was approved by the Human Research Ethics Committee of Isfahan University of Medical Sciences (IR.MUI.RESEARCH.REC.1398.014; Project Number: 397735).

\section{Phase I: DSMQ-SCT development}

\section{Item generation}

Item generation was carried out in Two steps:1) A review of broad literature relating to the SCT was conducted ${ }^{11,15,22,23}$ and 2 ) two focus group discussions were performed with 30 patients with T2DM. In this step, an item pool was extracted from the focus group discussion findings and the literature.Finally, 
30 items with 4 constructs )self- efficacy, social support, outcome expectations and outcome expectancy ( were generated that covered self-management aspects such as glucose management, dietary control, physical activity, and foot care. The items were measured on a Likert scale ranging from 1 to 4 ( $1=$ completely disagree to $4=$ completely agree) .

\section{Content validity}

Qualitative and quantitative content validity was conducted by ten experts ( health education, nursing, endocrinology and metabolism) in both qualitative and quantitative methods. To examine the qualitative content validity method, the experts were asked to evaluate the questionnaire based on grammatical criteria, using appropriate and correct words, applying correct and proper order of words in items, and employing appropriate scoring. The quantitative content validity of the questionnaire was evaluated by calculating the content validity ratio(CVR) and the content validity index (CVI) for the items. To calculate CVR, the expert panel was requested to score each item from 1 to 3 with a three -point Likert scale: "necessary", "useful but not necessary", and "unnecessary". Next, based on Lawshe's table, items with a CVR score of 0.62 or above were accepted ${ }^{24}$. To calculate the CVI based on Waltz and Bausell's recommendation ${ }^{25}$, the same experts were asked to examine the degree of " relevance", "clarity", and "simplicity"of each item based on a four-point Likert scale. Then, items with a score above 0.79 were considered satisfactory ${ }^{26}$.

\section{Test of the pre-final version}

The pre-test was performed to clarify the wording and ease of understanding of the final version(face validity). According to Beaton, the minimum sample size required for the pre-test is 30 people 27 .

Qualitative and quantitative methods were used to evaluate face validity. In the qualitative step, we asked 30 patients similar to the target population to assess each item for 'difficulty', 'relevancy', and 'ambiguity'. In the next step,the impact score of each item was calculated using a 5-point Likert scale. Impact scores equal to 1.5 or higher were accepted ${ }^{28}$.

\section{Phase II: Cross-sectional study}

\section{Classical item analysis}

Classical Item Analysis (CIA) measures the use of the mean, standard deviation, skewness, and Corrected Item-Total Correlation (CITC). Items with CITC of lower than 0.3 were eliminated. The minimal acceptable value for skewness was $1.96^{24}$.

\section{Construct validity}

The factorial structure of DSMQ-SCT was tested with a holdout method for cross-validation, and the total sample $(n=460)$ was randomly divided into two sub-samples. An exploratory factor analysis (EFA) was conducted on sample calibration ( $n=230)$ to explore the factor structure. The Kaiser-Meyer-Olkin (KMO) and Bartlett's Test of Sphericity were used to determine the sampling adequacy and the appropriateness of the data for factor analysis. Subsequently, principal component analysis with the varimax rotation was 
performed to extract latent factors. To measure the number of factors, we used an eigenvalue equal to one or above, and considered a cutoff point of at least 0.40 to determine variables loaded by each factor ${ }^{29}$. Then, a confirmatory factor analysis (CFA) was conducted on sample validation $(n=230)$ to validate the factor structure constructed through the calibration sample. Model fit was evaluated with the following fit criteria: chi-square value $(\chi 2)$, relative chi-square value $(\chi 2 / d f)$, and approximate error roots (RMSEA, standardized fit index (NFI), The value-added fitted index (IFI), the comparative fit index (CFI), and the Tucker-Lewis index (TLI) were used to evaluate the constructed model. When the fit met the following criterion, it was acceptable: CFI, TLI, IFI, and NFI $\geq 0.9$ and RMSEA $<0.08$ 30,31.

\section{Convergent validity}

Convergent validity was assessed by comparing DSMQ-SCT scores to the HbA1c value, using Pearson correlations.

\section{Reliability}

Internal consistency was estimated using the Cronbach's alpha coefficient. The value of 0.70 or above was considered acceptable ${ }^{32}$.

\section{Other measurements and statistical analysis}

In this study, quantitative and qualitative variables were expressed as mean (standard deviation (SD) and number (percent), The SPSS software Version 16 (SPSS Inc., Chicago, IL, USA) and Amos Graphic software Version 23 (Chicago, IL, USA) were used to conduct all statistical analyses.

\section{Results}

The participants' mean age was $60.8 y e a r s( \pm 10.31)$. The majority of participants were female $(70.7 \%)$, married $(90.0 \%)$ and had educational levels of primary and high school $(73.3 \%)$. The duration of diabetes was 13years $( \pm 8.37)$, and the average glycosylated hemoglobin $(\mathrm{HbA1c})$ was $8.2 \%( \pm 1.82)$. Table 1 presents the demographic and clinical characteristics of the sample. Table1 displays further information about the participants' socio-demographic characteristics.

\section{Content validity}

In the qualitative content validity the wordings of 5 items were modified according to the experts' opinions. In the quantitative phase, based on Lawshe's table, 7 items having scores lower than 0.62 were removed. Additionally, in the evaluation of CVI,based on Waltz and Bausell's index, all 23 items gained a score higher than 0.79 .

\section{Face validity}


In qualitative face validity, 3 items that were reported to be difficult to understand were modified . In quantitative face validity, 2 items that had gained the impact scores lower than 1.5 were removed. Hence, the questionnaire entered the construct validity phase with 23 items.

\section{Classical item analysis}

Ceiling and floor effects were observed in none of the items. CITC was higher than 0.3 for all items. The skewness of the items was less than 1.96, and no items were deleted in this step. Hence, 21 items were retained for the exploratory factor analysis.

\section{Exploratory factor analysis}

The Result of the Kaiser-Meyer-Olkin test $(K M O=0.89)$ and Bartlett's test of sphericity $(\chi 2=5850.855$; $\mathrm{P}<0.001$; $\mathrm{df}=210$ ) on 230 patients indicated that the data were appropriate for the factor analysis. The principal component analysis (PCA) with the varimax rotation was extracted from a four-factor solution for 21 items based on an eigenvalue greater than one. The extracted 4 factors explained $65.9 \%$ of the total variance. Table 2 shows the loading factor of each item after the varimax rotation.

\section{Confirmatory factor analysis}

The results of CFA showed a good fit for the data as follows: The relative chi-square ( $\chi 2 / \mathrm{df})$ was equal to 1.97, indicating the fitness of the model $(P<0.0001)$. The comparative indices of the model including $\mathrm{CFI}$ and IFI were higher than 0.9 (0.971 and 0.971, respectively) and NFI was equal to 0.942. the RMSEA of the model was 0.046 (confidence interval $90 \%=0.044-0.066$ ). Table 3 shows the results of CFA.

\section{Convergent validity}

Convergent validity indicated significant negative correlations between the DSMQ-SCT sub-scales and the HbA1c value $(P<0.001$, Table 5$)$.

\section{Reliability}

The Cronbach's alpha for the entire questionnaire was 0.88 and it ranged from 0.85 to 0.92 for the subscales. The results showed satisfactory internal consistency.

\section{Discussion}

This study aimed to develop and evaluate the psychometric properties of a new instrument to assess social cognitive factors associated with the self-management behavior in patients with type 2 diabetes. 
For some reasons, this study has a novel approach. First, there is strong evidence for the influence of social cognitive factors on the self-management behavior of patients with type 2 diabetes ${ }^{14,33-36}$. However ,there was no comprehensive theory-based instrument to assess the social cognitive factors of self-management behavior in patients with type 2 diabetes ${ }^{16,18}$. However, DSMQ-SCT is a multidimensional instrument designed based on the social cognitive theory (SCT) and fills this gap.Secondly, DSMQ-SCT can contribute to the development and assessment of theory-based interventions to improve self-management in patients with type 2 diabetes; it is also useful for practitioners in helping patients to improve or maintain good glycaemic control. The DSMQ-SCT evaluation indicates very good psychometric properties with satisfactory reliability and good validity. To design the instrument,first a preliminary 30-item inventory was developed based on the four constructs of SCT. Next, content and face validity were performed and 9 items were removed in this step. Afterward, 21 items were retained for the next step. Then, item analysis was performed and no items were removed in this step. Finally, 21 items were retained for the factor analysis. The KMO measure of sampling adequacy was 0.89 and Bartlett'stest of sphericity was significant $(p<0.001)$, indicating that the data are appropriate for factor analysis. No items were removed in this step, probably because this instrument was validated in previous steps.Principal component analysis with the varimax rotation resulted in a fourfactor structure. These four factors were able to explain $65.9 \%$ of the total variance. Based on exploratory factor analysis, four -factors, namely self-efficacy, social support, outcome expectations and outcome expectancy were extracted, which The first-factor extracted in the present study was outcome expectations. The first factor with a special value of 7.23 at $34.42 \%$ had the highest contribution to explaining the total variance. Outcome expectations are personal beliefs in the effect of an action on achieving a particular outcome. Outcome expectations can be positive and negative ${ }^{37}$. Positive outcomes expectancies include pleasurable sense experiences, social acceptance and support, being encouraging and receiving cash bonus and/or self-satisfaction .Furthermore, negative outcomes expectancies can include some cases like physical pain and many studies have demonstrated a significant association between outcome expectancy and diabetes self-management behaviors ${ }^{10,38-40}$. The second factor with a high variance in the exploratory factor analysis was the self-efficacy.Self-efficacy is one of the main concepts of the SCT theory and affects individuals' motivation while enforcing consistency in behavior. Self-efficacy is the confidence that individuals have in their ability to pursue a particular behavior. It has various sources, including one's accomplishments and failures, observance of the success or failure of others, and verbal persuasion ${ }^{41}$.Self-efficacy is the most important predictor of self-management behaviors in patients with diabetes ${ }^{23}$.Previous studies have shown that self-efficacy due to improve in diabetic Self-Management ${ }^{33}$.In this study, social support was the third important determinant of diabetic self-management.Social support has been interpreted as a sense of acceptance by others as well as enjoying the kindness, companionship, and attention of family members, friends, and others, so that family support has been expressed as a vital component of successful diabetes control. The relevant research literature reveals that low social support from friends and other members of the community can affect health status, and colleagues consider family support to be the strongest determining factor in following a treatment regimen in patients with type 2 diabetes and those receiving higher social support 
from their spouse and those around them, and they more committed to self-care behaviors ${ }^{34}$. Idalski et al. also reported that social support directly affected the control and improvement of self-care behaviors in patients with diabetes ${ }^{42}$.The last extracted factor was outcome expectancy.Outcome expectancy refers to the value a person places on the probable outcomes resulting from performing a behavior. The higher the expectancies, the higher the chances of indulging in the behavior ${ }^{43}$. Outcome expectancies is one important predictor of self-management in patients with diabetes ${ }^{38}$. CFA was applied to determine whether the hypothesized model was recognized from the EFA fit the data. The CFA results(the CFI =.971, $\mathrm{IFI}=.971, \mathrm{NFI}=.942, \mathrm{TLI}=.966$, the RMSEA $=.046$, and the relative chi-square $(\mathrm{x} 2 / \mathrm{df})=1.972$ ( confirmed that the data had a good fit with the four-factor scale. Furthermore,convergent validity indicated a negative and significant correlation between the total scale and subscales of the DSMQ-SCT and the $\mathrm{HbA1c}$ level.Glycosylated hemoglobin is regarded as the most important measure of long-term blood sugar control, and it is considered the gold standard of diabetes care.Previous studies indicated that social cognitive factors were associated with glycemic control in type 2 diabetes patients ${ }^{35,44,45}$. Good internal consistency of the total scale and its subscales was supported by a Cronbach's alpha coefficient of 0.88 or above.

\section{Strengths and Limitations}

To date, this is the first study to assessing the social cognitive factors of self-management in patients with type 2 diabetes based on the social cognitive theory. Our study has several strengths. (1) The glycemic level was determined based on the HbA1c laboratory test that had a high specificity ${ }^{46}$.(2) The DSMQ-SCT is a multidimensional and theory-based instrument covering the main aspects of diabetes self-management. (3) the DSMQ-SCT is user-friendly and due to the small number of questions, it can be completed in a short time.(4)The present study has provided good evidence regarding the construct validity and reliability of DSMQ-SCT.This study also had limitations: (1) We did not evaluate the testretest reliability. (2) The research data were collected only from patients who visited the Diabetes Clinic of the Isfahan Endocrine and Metabolism Research Center (in central Iran). Therefore, its findings could not be generalized to the entire population of type 2 diabetic patients in contexts. (3) In this study, we evaluated DSMQ-SCT only in patients with type 2 diabetes. Therefore, the examination of the validity and reliability of DSMQ-SCT among patients with type 1 diabetes provides an excellent opportunity to compare Social Cognitive factors associated with Self-Management among different types of diabetes. (4) One of the limitations of this study is that we did not measure discriminated validity, and we recommend measuring this type of validity in further studies.

\section{Conclusion}

In conclusion, our study findings provide preliminary support for DSMQ-SCT as a reliable, valid instrument for the assessment of social cognitive factors related to self-management. We maintain that DSMQ-SCT provides useful evidence for researchers and health professionals Accordingly, they can obtain a deeper and more accurate understanding of the social cognitive factors associated with the self- 
management behavior in patients with type 2 diabetes and are able to it use to assess effective theorybased interventions and to improve self-management in the growing population of patients with type 2 diabetes. However, more research needs to be conducted to confirm that DSMQ-SCT is a useful tool with sound psychometric properties.

\section{Abbreviations}

T2DM:Type 2 diabetes mellitus

IDF:International Diabetes Federation; MENA: Middle East and North Africa

SPSS: Statistical package for social science software package;

DSMQ-SCT: Diabetes Self-Management Questionnaire - social cognitive theory; EFA: Exploratory factor analysis; CFA: Confirmatory factor analysis; KMO: Kaiser-Meyer-Olkin test; PCA: Principal component analysis; the Classical Item Analysis (CIA) ; CFI: Comparative Fit Index; NFI: Normed Fit Index; IFI: Incremental Fit Index ; TLI : Tucker Lewis index ;X2: Model Chi-Square; RMSEA: Root Mean Square Error of Approximation.

\section{Declarations}

\section{Acknowledgments}

The findings of this study were part of a PhD thesis project (project number:397735). We acknowledge the contributions of Isfahan University of Medical Sciences, Isfahan, Iran for providing facilities to the study. Also, We appreciate the diabetic patients and all staff of Isfahan Endocrine and Metabolism Research for their excellent cooperation.

\section{Authors' contributions}

$A A E, M ~ S, H S$ and $H M$ contributed to the design and conception of the study. AA E and $H M$ performed the analysis and drafted the manuscript. $\mathrm{H} M$ wrote the manuscript and $A A E, M S$ and $H S$ contributed to the interpretation of data and revised the manuscript .All authors read and approved the final manuscript.

\section{Authors' information}

Hamed Mirzaei: Ph. D, Student, Health Education and Health

Promotion Department, School of Public Health, Isfahan University of Medical

Sciences, Isfahan, Iran.

Mansour Siavash: MD, Professor, Endocrinology \& Metabolism 
Department, School of Medicine, Isfahan University of Medical

Sciences, Isfahan, Iran.

Hossein Shahnazi: Ph.D, Associate Professor, Health Education and Health

Promotion Department, School of Public Health, Isfahan University of Medical

Sciences, Isfahan, Iran.

Ahmad Ali Eslami: Ph.D, Professor, Health Education and Health

Promotion Department, School of Public Health, Isfahan University of Medical

Sciences, Isfahan, Iran.

\section{Funding}

The study was supported by a grant from the Research and Technology

Deputy of Isfahan University of Medical Sciences. The funding body was only responsible for the cost of designing the study and analysis and interpretation of the data and did not have any role in writing the manuscript or publishing it in journals

\section{Availability of data and materials}

The data collection tools and datasets generated and/or analyzed during the current study are available from the corresponding author upon reasonable request.

\section{Ethics approval and consent to participate}

All participants received enough information about the study and Informed written consent was obtained from all participants. The study received ethical approval from the Ethics Committee of Isfahan University of Medical Sciences (IR.MUI.RESEARCH.REC.1398.014; Project Number: 397735) . 
Not applicable.

\section{Competing interests}

The authors declare that they have no competing interest.

\section{Author details}

${ }^{1}$ Student Research Committee and Department of Health Education and Health Promotion, School of Health, Isfahan University of Medical Sciences, Isfahan, Iran.

${ }^{2}$ Isfahan Endocrine and Metabolism Research Center, Isfahan University of Medical Sciences, Isfahan, Iran.

${ }^{3}$ Department of Health Education and Health Promotion, School of Health, zahedan University of Medical Sciences, zahedan, Iran.

\section{References}

1. Shaw JE, Sicree RA, Zimmet PZ. Global estimates of the prevalence of diabetes for 2010 and 2030. Diabetes Res Clin Pract. 2010;87(1):4-14. doi:10.1016/j.diabres.2009.10.007

2. Diabetes Federation International. IDF Diabetes Atlas 2019. 9th edn. IDF; 2019.

3. Mogre V, Johnson NA, Tzelepis F, Hall A, Paul C. Barriers to self-care and their association with poor adherence to self-care behaviours in people with type 2 diabetes in Ghana: A cross sectional study. Obes Med. 2020;18:100222. doi:10.1016/j.obmed.2020.100222

4. Ansari-Moghaddam A, Setoodehzadeh F, Khammarnia M, Adineh HA. Economic cost of diabetes in the Eastern Mediterranean region countries: A meta-analysis. Diabetes Metab Syndr Clin Res Rev. 2020;14(5):1101-1108. doi:https://doi.org/10.1016/j.dsx.2020.06.044

5. Simmons D, Lillis S, Swan J, Haar J. Discordance in perceptions of barriers to diabetes care between patients and primary care and secondary care. Diabetes Care. 2007;30(3):490-495.

6. American Diabetes Association. Standards of Medical Care for Patients With Diabetes Mellitus. Diabetes Care. 2003;26(suppl 1):s33 LP-s50. doi:10.2337/diacare.26.2007.S33

7. Dehvan F, Qasim Nasif F, Dalvand S, Ausili D, Hasanpour Dehkordi A, Ghanei Gheshlagh R. Self-care in Iranian patients with diabetes: A systematic review and meta-analysis. Prim Care Diabetes. 2021;15(1):80-87. doi:10.1016/j.pcd.2020.08.013

8. Strom JL, Egede LE. The impact of social support on outcomes in adult patients with type 2 diabetes: a systematic review. Curr Diab Rep. 2012;12(6):769-781. doi:10.1007/s11892-012-0317-0

9. Chlebowy DO, Garvin BJ. Social support, self-efficacy, and outcome expectations. Diabetes Educ. 2006;32(5):777-786. 
10. Karl FM, Holle R, Schwettmann L, Peters A, Laxy M. Time preference, outcome expectancy, and selfmanagement in patients with type 2 diabetes. Patient Prefer Adherence. 2018;Volume 12:1937-1945.

11. Safari M, Shojaei Zadeh D, Ghofranipour F, Heidarnia AR PHAA. Theories, models and methods of health education and health promotion. In: 4th ed. Publication of Sobhan; 2009:100-112.

12. Cosansu G, Erdogan S. Influence of Psychosocial Factors on Self-Care Behaviors and Glycemic Control in Turkish Patients With Type 2 Diabetes Mellitus. J Transcult Nurs. 2014;25(1):51-59. doi:10.1177/1043659613504112

13. Amer FA, Mohamed MS, Elbur Al, Abdelaziz SI, Elrayah ZA. Influence of self-efficacy management on adherence to self-care activities and treatment outcome among diabetes mellitus type 2. Pharm Pract. 2018;16(4).

14. Brown SA, García AA, Brown A, et al. Biobehavioral determinants of glycemic control in type 2 diabetes: A systematic review and meta-analysis. Patient Educ Couns. 2016;99(10):1558-1567. doi:10.1016/j.pec.2016.03.020

15. Jang $Y$, Yoo H. Self-management programs based on the social cognitive theory for Koreans with chronic disease: a systematic review. Contemp Nurse. 2012;40(2):147-159. doi:10.5172/conu.2012.40.2.147

16. Lu Y, Xu J, Zhao W, Han H-R. Measuring self-care in persons with type 2 diabetes: a systematic review. J Contin Educ Heal Prof. 2016;39(2):131-184.

17. Caro-Bautista J, Martín-Santos FJ, Morales-Asencio JM. Systematic review of the psychometric properties and theoretical grounding of instruments evaluating self-care in people with type 2 Diabetes Mellitus. J Adv Nurs. 2014;70(6):1209-1227. doi:10.1111/jan.12298

18. Caro-Bautista J, Martín-Santos FJ, Morales-Asencio JM. Systematic review of the psychometric properties and theoretical grounding of instruments evaluating self-care in people with type 2 diabetes mellitus. J Adv Nurs. 2014;70(6):1209-1227. doi:10.1111/jan.12298

19. Bijl J V, Poelgeest-Eeltink A V, Shortridge-Baggett L. The psychometric properties of the diabetes management self-efficacy scale for patients with type 2 diabetes mellitus. $J$ Adv Nurs. 1999;30(2):352-359.

20. Naderimagham S, Niknami S, Abolhassani F, Hajizadeh E, Montazeri A. Development and psychometric properties of a new social support scale for self-care in middle-aged patients with type II diabetes (S4-MAD). BMC Public Health. 2012;12(1):1-9.

21. Kellar SP, Kelvin EA. Munro's statistical methods for health care research. In: 6th ed. Wolters Kluwer Health/Lippincott Williams \& Wilkins; 2013:371-408.

22. Plotnikoff RC, Lippke S, Courneya KS, Birkett N, Sigal RJ. Physical activity and social cognitive theory: A test in a population sample of adults with type 1 or type 2 diabetes. Appl Psychol. 2008;57(4):628-643. doi:10.1111/j.1464-0597.2008.00344.x

23. Karimy M, Koohestani $H$, Araban $M$. The association between attitude, self-efficacy, and social support and adherence to diabetes self-care behavior. Diabetol I\& Metab Syndr. 2018;10.

24. Lawshe CH. A quantitative approach to content validity. J Pers Psychol. 1975;28(4):563-575. 
25. Waltz CF, Bausell RB. Nursing Research: Design, Statistics, and Computer Analysis. FA Davis Co; 1981.

26. Polit DF, Beck CT, Owen S V. Is the CVI an acceptable indicator of content validity? Appraisal and recommendations. Res Nurs Health. 2007;30(4):459-467. doi:https://doi.org/10.1002/nur.20199

27. Beaton DE, Bombardier C, Guillemin F, Ferraz MB. Guidelines for the process of cross-cultural adaptation of self-report measures. Spine J. 2000;25(24):3186-3191.

28. Bolarinwa OA. Principles and methods of validity and reliability testing of questionnaires used in social and health science researches. Niger Postgr Med J. 2015;22(4):195.

29. Hu L, Bentler PM. Fit indices in covariance structure modeling: Sensitivity to underparameterized model misspecification. Psychol Methods. 1998;3(4):424.

30. Tinsley HEA, Brown SD. Handbook of Applied Multivariate Statistics and Mathematical Modeling. Academic press; 2000.

31. Schreiber JB, Nora A, Stage FK, Barlow EA, King J. Reporting structural equation modeling and confirmatory factor analysis results: A review. J Educ Res. 2006;99(6):323-338.

32. Terwee CB, Bot SDM, de Boer MR, et al. Quality criteria were proposed for measurement properties of health status questionnaires. J Clin Epidemiol. 2007;60(1):34-42.

33. Mohebi S, Azadbakht L, Feizi A, Sharifirad G, Kargar M. Review the key role of self-efficacy in diabetes care. J Educ Health Promot. 2013;2:36.

34. Rad GS, Bakht LA, Feizi A, Mohebi S. Importance of social support in diabetes care. J Educ Health Promot. 2013;2:62. doi:10.4103/2277-9531.120864

35. Beckerle CM, Lavin MA. Association of self-efficacy and self-care with glycemic control in diabetes. Diabetes Spectr. 2013;26(3):172-178.

36. Gu L, Wu S, Zhao S, et al. Association of Social Support and Medication Adherence in Chinese Patients with Type 2 Diabetes Mellitus. Int J Environ Res Public Health. 2017;14(12). doi:10.3390/ijerph14121522

37. Schwarzer R, Lippke S, Luszczynska A. Mechanisms of health behavior change in persons with chronic illness or disability: the Health Action Process Approach (HAPA). Rehabil Psychol. 2011;56 3:161-170.

38. Wu S-FV, Courtney M, Edwards H, McDowell J, Shortridge-Baggett LM, Chang P-J. Self-efficacy, outcome expectations and self-care behaviour in people with type 2 diabetes in Taiwan. J Clin Nurs. 2007;16(11c):250-257.

39. Gibson B, Yingling L, Bednarchuk A, Janamatti A, Oakley-Girvan I, Allen N. An Interactive Simulation to Change Outcome Expectancies and Intentions in Adults With Type 2 Diabetes: Within-Subjects Experiment. JMIR Diabetes. 2018;3(1):e2.

40. Williams KE, Bond MJ. The roles of self-efficacy, outcome expectancies and social support in the self-care behaviours of diabetics. Psychol Health Med. 2002;7(2):127-141. 
41. Glanz K, Rimer BK, Viswanath K. Health behavior and health education: theory, research, and practice. In: 4th ed. John Wiley \& Sons; 2008:171-180.

42. Idalski Carcone A, Ellis DA, Weisz A, Naar-King S. Social support for diabetes illness management: supporting adolescents and caregivers. J Dev Behav Pediatr. 2011;32(8):581-590. doi:10.1097/DBP.0b013e31822c1a27

43. Sharma M. Theoretical Foundations of Health Education and Health Promotion. Jones \& Bartlett Learning; 2008.

44. Al-Khawaldeh OA, Al-Hassan MA, Froelicher ES. Self-efficacy, self-management, and glycemic control in adults with type 2 diabetes mellitus. J Diabetes Complications. 2012;26(1):10-16. doi:10.1016/j.jdiacomp.2011.11.002

45. Nozaki T, Morita C, Matsubayashi S, et al. Relation between psychosocial variables and the glycemic control of patients with type 2 diabetes: A cross-sectional and prospective study. Biopsychosoc Med. 2009;3.

46. Stubbs B, Vancampfort D, De Hert M, Mitchell AJ. The prevalence and predictors of type two diabetes mellitus in people with schizophrenia: a systematic review and comparative meta-analysis. Acta Psychiatr Scand. 2015;132(2):144-157.

\section{Tables}

Due to technical limitations, tables are only available as a download in the Supplemental Files section.

\section{Figures}




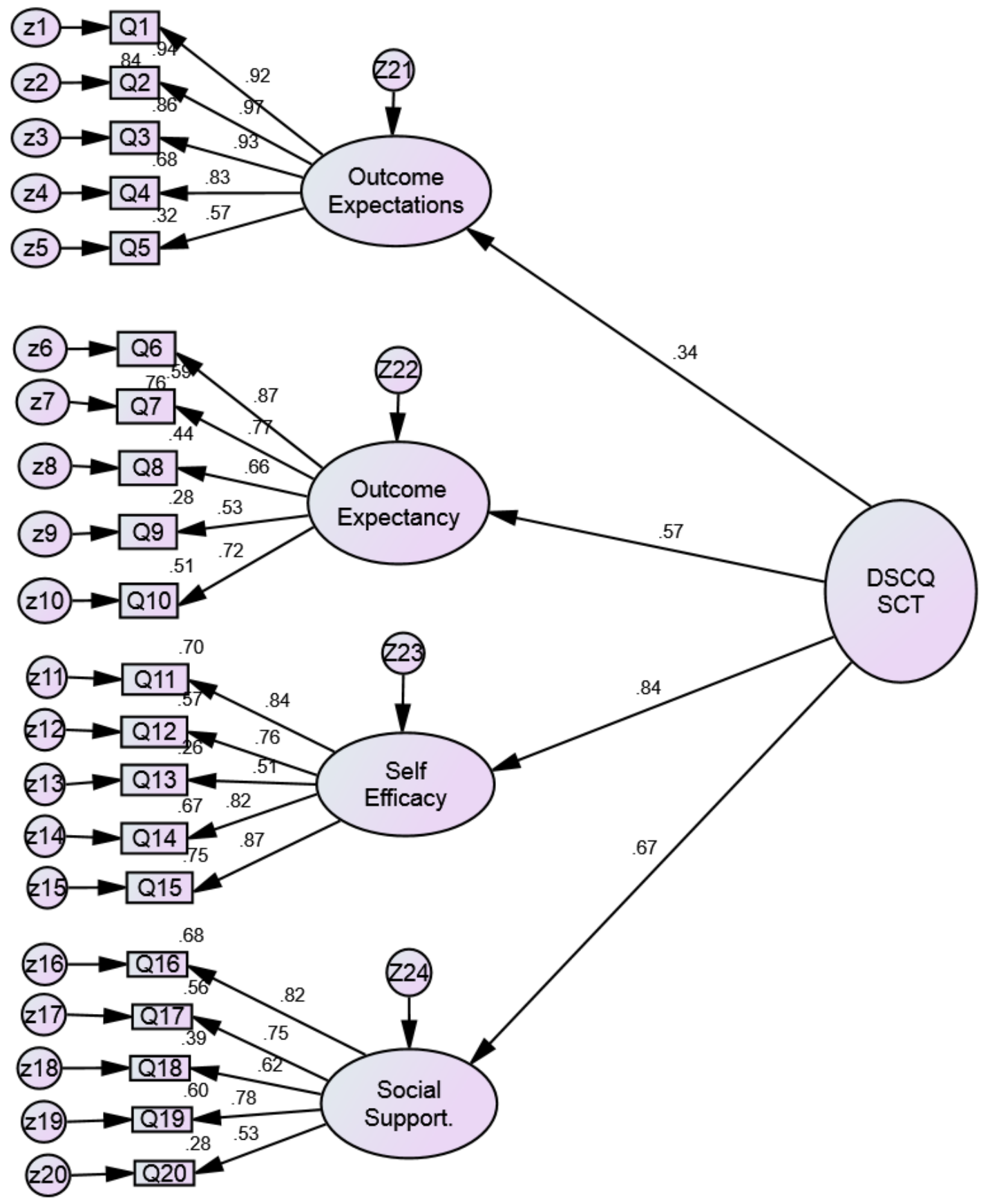

Figure 1

Confirmatory factor analysis to assess the measurement model of the DSMQ-SCT $(n=230)$

\section{Supplementary Files}

This is a list of supplementary files associated with this preprint. Click to download. 
- Tables.docx

Page $17 / 17$ 